Biosciences, MA, USA). In brief, EVs were captured and immobilized on ExoView Tetraspanin chips using the capture antibodies CD81, CD63, and CD9. Size was determined using SP-IRIS technology. Concentration measurements were performed with fluorescently labeled detection antibodies, specifically tetraspanins (CD81/CD63/CD9) for total EVs and CD54 and CD62E for specific EV populations.

Results: Analysis of EVs size confirmed the presence of small EVs (sEVs, < $70 \mathrm{~nm}$ ) in both study groups. The size of EVs did not vary significantly between study groups (mean size \pm SD in APS vs. HBD; CD81 (63.1 $\pm 1.5 \mathrm{~nm}$ vs. $66.6 \pm$ $10.6 \mathrm{~nm}), \mathrm{CD} 63(63.3 \pm 1.64 \mathrm{~nm}$ vs. $69.2 \pm 13.2 \mathrm{~nm})$, and CD9 capture spot $(61.8$ $\pm 1.18 \mathrm{~nm}$ vs. $64.1 \pm 7.64 \mathrm{~nm}$ )). The levels of total EVs (tetraspanin-positive) were increased 1.7-fold, 1.4-fold, and 2.2-fold for CD81, CD63, and CD9 capture spots, respectively, in APS patients compared to HBDs (Figure 1A). In addition, CD54and CD62E-positive EVs represented a small population $(<2 \%)$ of the total EVs (Figure 1B). The levels of CD54 were increased 2.9-fold, 3.0-fold, and 2.5-fold on CD81, CD63, and CD9 capture spots, respectively, and similarly, the levels of CD62E were increased 2.2-fold, 2.7-fold, and 2.0-fold on CD81, CD63, and CD9 capture spots, respectively, in APS patients compared to HBDs (Figure 1A).

A
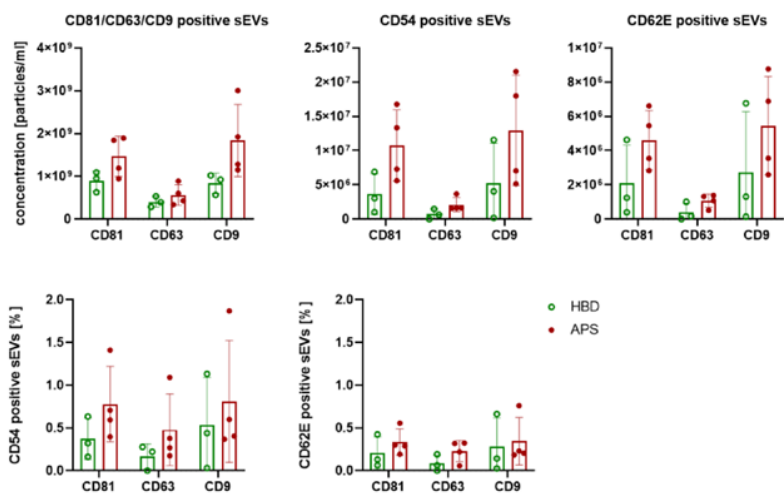

Capture spot

Figure 1. Concentration of tetraspanin- (CD81/CD63/CD9), CD54- and CD62- positive sEVs in APS patients and HBDs.

Conclusion: Higher levels of SEVs and increased percentage of CD54- and CD62E-positive sEVs in plasma of APS patients could indicate an altered and activated endothelium in those patients.

REFERENCES:

[1] Miyakis S. Journal of thrombosis and haemostasis. 2006.

[2] Zara M. Int J Mol Sci. 2019.

[3] Chaturvedi S. Semin Thromb Hemost. 2018.

[4] Stok U. Cells. 2020.

[5] Oggero S. Front Pharmacol. 2019.

Disclosure of Interests: Ula Stok: None declared, Alex Shephard Employee of: NanoView Biosciences., Sasa Cucnik: None declared, Snežna Sodin-Šemrl: None declared, Polona Zigon: None declared

DOI: 10.1136/annrheumdis-2021-eular.1371

\section{AB0081 ENDOTHELIAL MARKERS WITH DYSREGULATION IN SYSTEMIC LUPUS ERYTHEMATOSUS: A SYSTEMATIC LITERATURE REVIEW}

S. Bergkamp ${ }^{1}$, M. J. Wahadat ${ }^{2}$, V. Smith ${ }^{3,4,5}$, S. Kamphuis ${ }^{2}$, J. M. Van den Berg ${ }^{1}$, D. Schonenberg-Meinema ${ }^{1}{ }^{1}$ Amsterdam UMC, Paediatric Rheumatology, Amsterdam, Netherlands; ${ }^{2}$ Erasmus MC, Paediatric Rheumatology, Rotterdam, Netherlands; ${ }^{3}$ Ghent University, Internal Medicine, Gent, Belgium; ${ }^{4}$ Ghent University Hospital, Rheumatology, Gent, Belgium; ${ }^{5} \mathrm{VIB}$ Inflammation Research Centre (IRC), Unit for Molecular Immunology and Inflammation, Ghent, Belgium

Background: Systemic lupus erythematosus (SLE) is a severe, lifelong autoimmune disease known for its multisystem organ involvement. SLE patients are known to be at risk for premature atherosclerosis at a relatively young age (1). Endothelial dysregulation is one of the pathophysiologic mechanisms that can lead to the higher risk for cardiovascular disease in SLE (2). Multiple endothelial markers with dysregulation in SLE have been described so far, of which some are associated with disease activity.

Objectives: To report a systematic literature review regarding endothelial markers that are dysregulated in SLE and search for associations with disease activity. Methods: The search was performed according to the Preferred Reporting Items for Systematic review and Meta-analysis Protocols (PRISMA-P) 2015 (3). In
July 2020, the search terms were used in Embase, MEDLINE, Web of Science, Google Scholar and Cochrane. Inclusion criteria were 1) published studies after the year 2000 that reported measurements of endothelial cell markers in serum and/or plasma of SLE patients (diagnosed according to ACR/SLICC criteria), 2) English language peer reviewed articles and 3) disease activity measurement (i.e. SLEDAI, BILAG, SLAM, ECLAM or PGA). Exclusion criteria were 1) case reports or editorials, 2) studies performed in animals and 3) studies with microRNA/cytokine biomarkers. There was no minimum count for study population. The screening process is shown in figure 1 .

Results: From 1892 hits, we identified 110 eligible articles. Table 1 shows an overview of the most frequently studied endothelial markers. These identified endothelial markers are involved in endothelial cell (EC) activation, EC apoptosis, disturbed angiogenesis, defective vascular tone control, immune dysregulation and coagulopathy. In most studies, dysregulation of the endothelial marke was associated with disease activity. The majority of the studies had a cross-sectional design, longitudinal data on endothelial markers in SLE are scarce.

Conclusion: We identified multiple endothelial markers that are dysregulated in SLE and this dysregulation was often associated with disease activity in cross-sectional studies. Our future plan is to test the identified endothelia markers in (longitudinally collected) samples of (childhood onset) SLE patients, disease- and healthy controls. This will be a next step in unravelling the pathophysiology of premature atherosclerosis and cardiovascular events in SLE-patients in young adulthood.

Table 1.

\begin{tabular}{|c|c|c|c|c|c|c|c|}
\hline \multirow[t]{2}{*}{$\begin{array}{l}\text { Endothelial } \\
\text { marker }\end{array}$} & \multirow[t]{2}{*}{$\begin{array}{l}\text { Articles } \\
\text { (no. of } \\
\text { studies) }\end{array}$} & \multicolumn{3}{|c|}{$\begin{array}{l}\text { Significant } \\
\text { correlation with } \\
\text { SLEDAl } \\
\text { (no. of studies) }\end{array}$} & \multicolumn{2}{|c|}{$\begin{array}{l}\text { Studies with control groups } \\
\text { (no. of studies) }\end{array}$} & \multirow[t]{2}{*}{$\begin{array}{l}\text { Longitudinal } \\
\text { data } \\
\text { (no. of } \\
\text { studies) }\end{array}$} \\
\hline & & Yes & & Unknown & $\begin{array}{l}\text { Healthy } \\
\text { controls }\end{array}$ & Diseased controls & \\
\hline VCAM-1 & 26 & 20 & 1 & 5 & 23 & 3 & 8 \\
\hline VEGF & 22 & 13 & 5 & 4 & 20 & 2 & 2 \\
\hline ICAM-1 & 18 & 11 & 5 & 2 & 17 & 3 & 4 \\
\hline Thrombomodulin & 17 & 10 & 5 & 2 & 13 & 2 & 2 \\
\hline E-Selectin & 13 & 6 & 5 & 2 & 10 & 2 & 1 \\
\hline MCP-1 & 8 & 6 & 2 & 0 & 7 & 1 & 2 \\
\hline P-Selectin & 6 & 3 & 2 & 1 & 6 & 1 & 1 \\
\hline IP-10 & 6 & 5 & 1 & 0 & 5 & 0 & 1 \\
\hline Pentraxin-3 & 5 & 5 & 0 & 0 & 5 & 1 & 2 \\
\hline vWF & 5 & 4 & 1 & 0 & 5 & 0 & 2 \\
\hline Neopterin & 3 & 2 & 1 & 0 & 2 & 0 & 2 \\
\hline Fas & 3 & 0 & 2 & 1 & 2 & 0 & 1 \\
\hline Angiopoeietin-2 & 3 & 3 & 0 & 0 & 2 & 1 & - \\
\hline Endothelin-1 & 3 & 1 & 2 & 0 & 3 & 0 & - \\
\hline PAI-1 & 3 & 1 & 1 & 1 & 2 & 0 & - \\
\hline Adrenomedullin & 3 & 2 & 0 & 1 & 2 & 0 & - \\
\hline TWEAK & 3 & 1 & 1 & 1 & 1 & 2 & - \\
\hline PECAM-1 & 3 & 1 & 1 & 1 & 3 & 0 & - \\
\hline
\end{tabular}

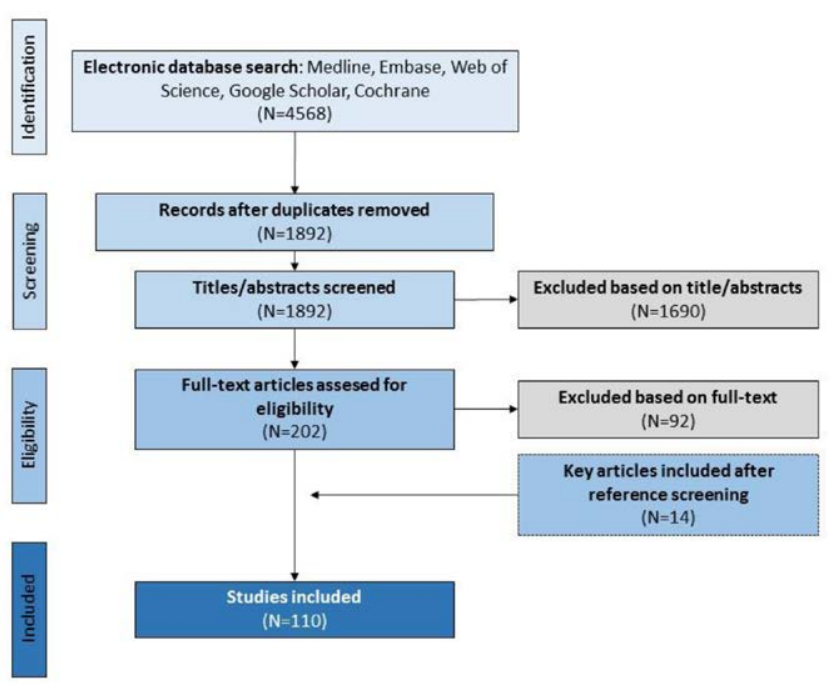

Figure 1.

REFERENCES:

[1] Vavlukis M, Pop-Gjorceva D, Poposka L, Sandevska E, Kedev S. Myocardial Infarction in Systemic Lupus Erythematosus - the Sex Specific Risk Profile. Curr Pharm Des. 2020 Dec 9. 
[2] Westerweel PE, Luyten RK, Koomans HA, Derksen RH, Verhaar MC. Premature atherosclerotic cardiovascular disease in systemic lupus erythematosus. Arthritis Rheum. 2007 May;56(5):1384-96.

[3] Shamseer L, Moher D, Clarke M, Ghersi D, Liberati A, Petticrew M, Shekelle P, Stewart LA; PRISMA-P Group. Preferred reporting items for systematic review and meta-analysis protocols (PRISMA-P) 2015: elaboration and explanation. BMJ. 2015 Jan 2;350:g7647.

Disclosure of Interests: None declared

DOI: 10.1136/annrheumdis-2021-eular.2008

\section{AB0082 PERSISTENT LOW COMPLEMENT LEVELS AND INTER FERON GENE UPREGULATION ARE PREDICTIVE FOR DISEASE PROGRESSION IN PATIENTS WITH INCOM- PLETE SYSTEMIC LUPUS ERYTHEMATOSUS}

W. Lambers ${ }^{1}$, J. Westra ${ }^{2}$, S. Arends ${ }^{2}$, B. Doornbos- van der Meer ${ }^{2}$, B. Horvath ${ }^{3}$, H. Bootsma ${ }^{2}$, K. De Leeuw ${ }^{2} .{ }^{1}$ University Medical Centre Groningen, Rheumatology/Clinical Immunology, Groningen, Netherlands; ${ }^{2}$ University Medical Center Groningen, Rheumatology/Clinical Immunology, Groningen, Netherlands; ${ }^{3}$ University Medical Center Groningen, Dermatology, Groningen, Netherlands

Background: A subgroup of lupus patients present with mild symptoms and immunologic features, while they do not meet classification criteria for SLE. This disease state can be referred to as "incomplete systemic lupus erythematosus" (iSLE). Up to $55 \%$ of iSLE patients progress to SLE. Furthermore, previous research has shown that iSLE might overlap with early primary Sjögren's disease (pSS).(1) Unfortunately, there are no predictive markers available for progression to classifiable disease. Type-l interferon (IFN) plays an important role in disease initiation of both SLE and pSS. $(2,3)$ Myxovirus-resistance protein A $(M \times A)$ is a GTP-ase that has previously be demonstrated to correlate strongly with IFN-type I expression. Furthermore, interferon-inducible chemokines IFN- $\gamma$ induced protein 10 (IP-10), and B-cell activating factor (BAFF), that are both inducible by IFN, are of interest, because it is demonstrated that these proteins are increased prior to the diagnosis of SLE.(4)

Objectives: To find predictive markers that identify patients with incomplete systemic lupus erythematosus (iSLE) who are at the highest risk to progress to classifiable systemic lupus erythematosus (SLE) or primary Sjögren's syndrome (pSS).

Methods: Patients with iSLE (ANA $\geq 1: 80, \geq 1$ clinical SLICC criterion, but not fulfilling the criteria, and disease manifestation $<5$ years) were included in a longitudinal observational study. Every half year, clinical status was evaluated and regular immunological serologic assessment was performed. Annually, interferon (IFN)-gene expression was determined by RT-PCR in whole blood using 14 genes. These genes represented 3 IFN-related modules. Some genes were mainly inducible by IFN-type I, others by IFN-type II. Furthermore, IFN-related mediators Myxovirus resistance protein A (MxA), interferon-gamma-induced protein 10 (IP-10) and B-cell activating factor (BAFF) were measured.

Results: Of 38 included iSLE patients, 6 had developed SLE and 1 develop pSS $(18 \%)$ after median follow up of 36 months. The 7 patients who developed SLE/pSS were all women, and were younger at baseline than those who remained having iSLE (median 26 years, IQR $20-29$ vs. median 42 years, IQR $30-56, p=0.0009)$. Over time, these patients had significantly lower complement $3(p<0.0001)$ and complement 4 levels $(p=0.005)$, higher IFN-gene expression $(p=0.007)$, and lower neutrophil counts $(p=0.033)$ (see Figure 1.). No difference was found between IFN-type I and IFN-type II inducible genes. Levels of MxA, IP-10 and BAFF did not differ between patients who remained iSLE and who progressed to SLE/pSS.

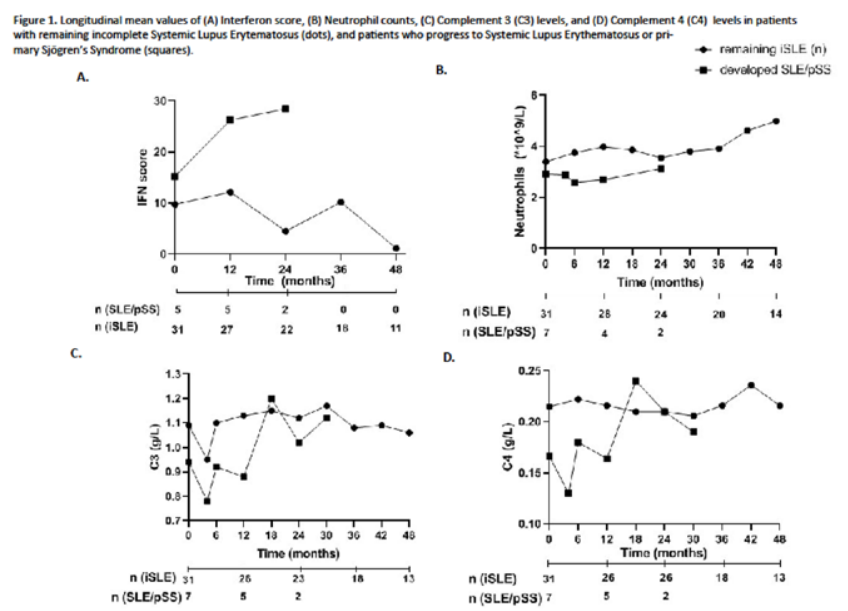

Figure 1.
Conclusion: Gender, age at diagnosis, persistent low complement levels, and high IFN-gene expression can help to identify iSLE patients at the highest risk of progressing to classifiable disease.

\section{REFERENCES:}

[1] Md Yusof MY, et al. Prediction of autoimmune connective tissue disease in an at-risk cohort: Prognostic value of a novel two-score system for interferon status. Ann Rheum Dis. 2018;1-8.

[2] Yao Y, et al. Type I interferons in Sjögren's syndrome. Autoimmun Rev. 2013;12(5):558-66.

[3] Crow MK. Type I Interferon in the Pathogenesis of Lupus. J Immunol [Internet]. 2014;192(12):5459-68.

[4] Lu R, et al. Dysregulation of innate and adaptive serum mediators precedes systemic lupus erythematosus classification and improves prognostic accuracy of autoantibodies. J Autoimmun. 2016;74:182-93.

Disclosure of Interests: None declared

DOI: 10.1136/annrheumdis-2021-eular.2306

\section{AB0083 SIGNALING OF PSGL-1 IN HUMAN NEUTROPHILS MIGHT CONTROL NET GENERATION AND APOPTOSIS AND MIGHT BE ALTERED IN SLE PATIENTS}

A. Muñoz-Callejas ${ }^{1}$, E. González Sánchez ${ }^{1}$, E. F. Vicente-Rabaneda ${ }^{2}$, J. Garcia Perez $^{3}$, S. Castañeda ${ }^{2}$, A. Urzainquii . 'Instituto de Investigación SanitariaHospital de la Princesa, Inmunologia, Madrid, Spain; ${ }^{2}$ Hospital de la Princesa, Reumatologia, Madrid, Spain; ${ }^{3}$ Hospital de la Princesa, Neumologia, Madrid, Spain

Background: Neutrophil extracellular traps (NETs) are extracellular chromatin fibers decorated with neutrophil antimicrobial proteins and histones, which are formed by neutrophils to trap invading pathogens and facilitate their removal. Excessive presence of NETs has been reported in autoimmune diseases like Systemic Lupus Erythematosus (SLE) and has been related with disease pathogenesis [1]. Our previous work showed that P-selectin KO mice develop an autoimmune syndrome similar to human lupus and that patients with cutaneous lupus have reduced expression of P-selectin in skin vessels [2]. Although it has been reported that $P$-selectin might induce NET generation in mouse neutrophils [3] there are no studies performed with human neutrophils.

Objectives: 1)To analyze the contribution of PSGL-1/P-Selectin interaction to control the generation of NETs by human neutrophils from healthy donors and patients with SLE.

2)To study the implication of PSGL-1/P-Selectin interaction in the control of other types of neutrophil death.

Methods: Human neutrophils were isolated from healthy donors and patients with SLE. After incubation in vitro with BSA or P-Selectin in rolling-like conditions (60 rpm shaking at $37^{\circ} \mathrm{C}$ ) for $10 \mathrm{~min}$, neutrophils were left to adhere for 1 hour. Then, NETs were labeled with Sytox Green and quantified by fluorimetry and by flow cytometry (FACSCanto II, BD Biosciences). For quantification of necrosis and apoptosis, cells were labeled with propidium iodide and annexing $\mathrm{V}$ and analyzed by flow cytometry. Data were analyzed with two-sided Student, twosided Mann-Whitney U or two-sided Wilcoxon signed rank test using GraphPad Prism software (Version 8.0.1, La Jolla, CA). Results were considered significant at $\mathrm{P}<.05$.

Results: Our results show that rolling on BSA, compared to adhesion, induces NET generation and apoptosis of neutrophils isolated from blood of healthy donors. Rolling on P-selectin reduces apoptosis of neutrophils and increase the percentage of NET events, although reducing NET intensity. Comparing to healthy donors, neutrophils from active SLE patients generate NETs with higher intensity in adhesion and do not respond to P-Selectin.

Conclusion: The interaction of PSGL-1 with P-Selectin preserves neutrophil death from apoptosis and controls NET generation. Our results suggest that this control might be altered in patients with active SLE.

REFERENCES:

[1] Fousert, E., Toes, R., \& Desai, J. (2020). Neutrophil extracellular traps (NETs) take the central stage in driving autoimmune responses. Cells, 9(4), 915.

[2] González-Tajuelo, R., Silván, J., Pérez-Frías, A., De La Fuente-fernández, M., Tejedor, R., Espartero-Santos, M. \& Urzainqui, A. (2017). P-Selectin preserves immune tolerance in mice and is reduced in human cutaneous lupus. Scientific reports, 7(1), 1-12.

[3] Etulain, J., Martinod, K., Wong, S. L., Cifuni, S. M., Schattner, M., \& Wagner, D. D. (2015). P-selectin promotes neutrophil extracellular trap formation in mice. Blood, 126(2), 242-246.

Disclosure of Interests: None declared

DOI: 10.1136/annrheumdis-2021-eular.2508 